\title{
Optimal Signal Processing of Frequency-Stepped CW Radar Data
}

\author{
Gary A. Ybarra, Member, IEEE, Shawkang M. Wu, Student Member, IEEE, Griff L. Bilbro, \\ Sasan H. Ardalan, Member. IEEE. Chase P. Hearn, and Robert T. Neece
}

\begin{abstract}
An optimal signal processing algorithm is derived for estimating the time delay and amplitude of each scatterer reflection using a frequency-stepped $C W$ system. The channel is assumed to be composed of abrupt changes in the reflection coefficient profile. The optimization technique is intended to maximize the target range resolution achievable from any set of frequency-stepped $\mathrm{CW}$ radar measurements made in such an environment. The algorithm is composed of an iterative two-step procedure. First, the amplitudes of the echoes are optimized by solving an overdetermined least squares set of equations. Then, a nonlinear objective function is scanned in an organized fashion to find its global minimum. The result is a set of echo strengths and time delay estimates. Although this paper addresses the specific problem of resolving the time delay between the first two echoes, the derivation is general in the number of echoes. Performance of the optimization approach is illustrated using measured data obtained from an HP-8510 network analyzer. It is demonstrated that the optimization approach offers a significant resolution enhancement over the standard processing approach that employs an IFFT. Degradation in the performance of the algorithm due to suboptimal model order selection and the effects of additive white Gaussion noise are addressed.
\end{abstract}

\section{INTRODUCTION}

$\mathbf{T}$ HE physical data measured by a monostatic frequencystepped CW (FSCW) radar system is a sequence of complex reflection coefficients as seen from the aperture plane to which the system is calibrated. The sequence of measured reflection coefficients may be interpreted as samples of the radar channel frequency response. Given these measurements, the objective is to determine the target range. The standard approach [1] is to perform an IFFT (Inverse Fast Fourier Transform) that produces an estimate of the radar channel time domain impulse response. Peaks in the impulse response correspond to reflections and their time delay corresponds to the range to the source of reflection. The resolution of this approach is limited by the measurement bandwidth. In certain applications, such as re-entry plasma density measurement [2], [3], the bandwidth must be kept small. The resolution offered

Manuscript received August 13, 1992, revised March 16, 1994. This work was supported in part by the National Acronatic, and Space Administration Langley Research Center under Grant NAG-1-1219.

G. A. Ybarra is with the Departnkent of Etectrical Engincering, Duke University, Durham, NC 27708 USA

S. M. Wu and G. I. Bilbro are with the Department of Electrical and Computer Engineering, North Carolina State Unversity, Raleigh, NC 27695 USA.

S. H. Ardatan is with X(AD) (orporation, (ary, NC 27511 USA.

C. P. Hearn and R. T. Neece are with NASA langley Revearch Center, Hampton, VA 23665 USA.

IEEE Log Number 9406826 by the IFFT approach is often unsatisfactory and an alternative approach is required. Even if there exists no distinct bandwidth constraint imposed by the radar channel, maximization of the achievable resolution for a particular measurement bandwidth is an important problem.

The FSCW measurement system is an outgrowth of Frequency-Modulated CW (FMCW) radar [4] that was well established in the 1970's and applied in several measurement environments such as locating buried PVC pipe |5|, subsurface water [6], underground tanks. and the level of liquid within them [7]. In these HMCW systems, a sinusoid is transmitted whose frequency is swept linearly with time. The differential frequency between the transmitted and received signals is converted to a range estimate. An FSCW system transmits a sequence of sinusoids at different frequencies and measures the steady-state amplitude and phase shift induced by the radar channel at each discrete frequency. One significant benefit of performing the measurements at discrete frequencies is the ease with which digital signal processing techniques may be applied to the data. With the availability of relatively low-cost digitally controlled oscillators. FSCW systems have been the focus of much attention in recent years. In 1982, Gjessing et al. [8] presented results combining the data produced by the analog FMCW and digital FSCW systems in the context of adaptive delection and identification of low-flying aircraft in a sea clutter backround using a matched filter approach. In 1990, Van Hanme [9] presented an FSCW technique based upon recursively minimizing a nonlinear objective function through approximation using a Taylor series expansion and demonstrated its performance through range detection of transmission line discontinuities. In 1991, Maricevic et al. [10] demonstrated that the Matrix Pencil algorithm developed by Hua et al. [11] could be applied to FSCW measurements of a transmission line having step discontinuities in characteristic impedance with significant resolution enhancement over standard IFFT processing. In 1992. Carriere and Moses [12] applied a total least squares Prony Estimator to FSCW measurements of scale models of commercial aircraft and demonstrated a significant enhancement in characterizing radar signatures over the Inverse Discrete Fourier Transform.

This paper presents the derivation of an optimal signal processing algorithm for obtaining the parameters of a propagation channel model. The extracted channel parameters are the round-trip time delay and amplitude of each reflection. The channel is assumed to be composed of abrupt changes in the reflection coefficient profile. The optimization technique 
is intended to maximize the target range resolution achievable from any set of frequency-stepped $\mathrm{CW}$ radar measurements made in such an environment. The derivation is general in the number of echoes, and a constant frequency interval between the measurement samples is not required. However, in order to illustrate the performance of the optimal processing algorithm through its geometric interpretation, the specific problem of resolving the time delay between the first two echoes is addressed. It will be shown that the optimization process can be interpreted as scanning a nonlinear objective function surface in an organized procedure to find its absolute minimum. In the two-reflection problem, the objective function surface is 3-dimensional, allowing visual inspection. By visually examining the structural change in these 3-D surfaces as measurement parameters are changed, significant insight into the underlying physical process becomes available at a glance.

The resolution limitations of the IFFT approach are demonstrated along with the enhancement offered by the optimal processing algorithm using physical measurements made with an HP-8510 network analyzer. Several sets of measured data are analyzed to illustrate the effects of varying bandwidth, target range, model order, and noise level. The first data set analyzed is a sequence of 801 reflection coefficients measured at the input port of the $7-\mathrm{mm}$ Beatty Standard [13] over a bandwidth of $18 \mathrm{GHz}$. The IFFT performs well in this case, clearly resolving two known primary reflections with the relatively large measurement bandwidth. The optimization approach in one delay dimension is then applied to this same data set to illustrate that "correct" model order selection is not required for accurately measuring the range to multiple sources of reflection. The effects of severe additive white gaussian noise are examined. It is shown that noise causes a decrease in the depth of the objective function minima as well as a perturbation in their location.

In order to illustrate the resolution enhancement achievable by optimal processing over the IFFT, the measurement bandwidth is reduced from 18 to $2 \mathrm{GHz}$. With $2-\mathrm{GHz}$ bandwidth, the IFFT approach clearly fails to resolve two distinct reflections that are known to exist. The optimal processing algorithm is then applied (in two delay dimensions) to the reduced bandwidth data and the results reveal the potential resolution achievable. The remaining data sets are extracted from measurements made at the input port of a test apparatus composed of a dielectric interferer and metal plate target, also driven by an HP-8510 network analyzer. Results for these data sets are similar to those presented for the Beatty Standard.

This paper is organized as follows. In the next section the optimal processing algorithm is derived in detail. The algorithm is formulated in the frequency domain and an iterative procedure is outlined that decomposes the problem into a sequence of linear and nonlinear minimization problems. Then, experimental data is presented and analyzed using both the standard IFFT and optimization approaches, and their performance is compared. Finally, the last section presents conclusions that may be drawn from the experimental results, and future research directions including unresolved problems are presented.

\section{DERIVATION OF THE OPTIMAL Signal Processing Algorithm}

Given a set of frequency response measurements generated by an FSCW radar system, the objective is to determine the target range. This may be accomplished by assuming an underlying physical model for the channel, computing the frequency response of the model at the frequencies at which the measurements were taken, and finally minimizing the norm of the difference between the physical measurements and the values produced by the model. The analytical expression for the objective function to be minimized, denoted by $J$, is given by

$$
J=\left\|H\left(j\left(\omega_{0}+i \Delta \omega\right)\right)-H_{m}\left(j\left(\omega_{0}+i \Delta \omega\right)\right)\right\|
$$

where the $H_{m}\left(j\left(w_{0}+i \Delta \omega\right)\right)$ are the measured complex reflection coefficient pairs, the $H\left(j\left(w_{0}+i \Delta \omega\right)\right)$ are the values of a theoretical model, $\|\cdot\|$ denotes vector norm [14], and $i$ is the index number of each measurement which spans the integer range from $1-n . J$ is a function of the parameters of the physical model yet to be specified.

The physical propagation path that will be assumed contains a highly reflective target, possibly embedded in an inhomogeneous medium. The inhomogeneity may be either continuously tapered, or composed of distinct media layers each having a different permittivity, or any combination of the two. The simplest realistic model for the system impulse response has the form

$$
h(t)=A_{1} \delta\left(t-t_{1}\right)+A_{2} \delta\left(t-t_{2}\right)+A_{3} \delta\left(t-t_{3}\right)+\cdots
$$

where the $A_{i}$ are the reflection amplitudes and the $t_{i}$ are the time delays to the reflections. The corresponding frequency response of the system is obtained by taking the Fourier Transform of (2), which produces

$$
H(j \omega)=A_{1} e^{-j \omega t_{1}}+A_{2} e^{-j \omega t_{2}}+A_{3} e^{-j \omega t_{3}}+\cdots
$$

Samples of this frequency frequency response form the model values comprising $H\left(j\left(\omega_{0}+i \Delta \omega\right)\right)$ in (1).

$$
\begin{aligned}
H\left(j\left(\omega_{0}+i \Delta \omega\right)\right)= & A_{1} e^{-j\left(\omega_{0}+i \Delta \omega\right) t_{1}}+A_{2} f^{-j\left(\omega_{0}+i \Delta \omega\right) t_{2}} \\
& +A_{3} e^{-j\left(\omega_{0}+i \Delta \omega\right) t_{3}}+\cdots
\end{aligned}
$$

Therefore, using the assumed model for the system impulse response (2), the objective function $J,(1)$ is a function of the reflection amplitudes and delays.

Using the 2-norm as the metric in (1), the general least squares optimization problem becomes finding the set of amplitudes and delays in the model that minimize

$$
J=\sum_{i=1}^{n}\left|H\left(j\left(\omega_{0}+i \Delta \omega\right)\right)-H_{m}\left(j\left(\omega_{0}+i \Delta \omega\right)\right)\right|^{2}
$$

where the summation index $i$ ranges over the $n$ measured data pairs. The objective is to find the global minimum of the performance metric (5) with respect to the amplitude-delay pairs $A_{i}, t_{i}$ in the model (2), (3). This objective is achieved by decomposing the performance metric (5) into a sum of linear and nonlinear components. The linear portion of the objective is a set of simultaneous linear equations whose solution yields 
the optimal amplitudes. The metric (5) is quadratic in the amplitude parameters $A_{i}$. This allows the $A_{i}$ to be calculated in closed form by solving the least squares (LS) problem

$$
F a \cong f
$$

where the complex $(n \times N)$ matrix $F$ is composed of frequency response estimates based on the model (4) and is given by (7), shown at the bottom of the page. $n$ is the number of frequency measurements and $N$ is the number of $A_{i}, t_{i}$ pairs to be extracted. The complex vector $f$ is composed of the physical frequency response measurements and is given by

$$
\boldsymbol{f}=\left[H_{M}\left(\omega_{1}\right) H_{M}\left(\omega_{2}\right) H_{M}\left(\omega_{3}\right) \cdots H_{M}\left(\omega_{n}\right)\right]^{T}
$$

The vector $\boldsymbol{a}$ in (6) contains the set of amplitudes $A_{i}$ to be determined

$$
\boldsymbol{a}=\left[\begin{array}{lllll}
A_{1} & A_{2} & A_{3} & \cdots & A_{N}
\end{array}\right]^{T}
$$

Solution of the least squares problem requires assuming a set of delays $t_{i}$. It will be shown that by "scanning "a sequence of amplitude optimized objective function values for certain delay combinations, it is possible to find the global minimum of (5). Finding the global, or absolute minimum of (5) determines the optimal set of reflection amplitude-delay pairs. If there exists any a priori knowledge about the target range such as upper and/or lower bounds, the range of the time delays over which the objective function is scanned can be limited and the amount of computation necessary to find the global minimum reduced. Placing bounds on the time scan of the objective function corresponds to time-gating, a procedure often implemented in conventional pulsed radar [16]-[21].

The solution to (5) will yield a set of complex amplitudes because the model (2-4) will not fit the physical data exactly. However, in the derivation which follows, the amplitude vector a is constrained to be real. The same approach that is used to solve the standard real LS problem using the normal equation approach [14], $|15|$ can be extended to solve this problem. The solution to (5) is found by minimizing the real scalar that results from the following squared inner product.

$$
\begin{aligned}
y= & \left\|\left(\boldsymbol{F}_{r}+j \boldsymbol{F}_{i}\right) \boldsymbol{a}-\left(\boldsymbol{f}_{r}+j \boldsymbol{f}_{i}\right)\right\|_{2}^{2} \\
y= & \left(\boldsymbol{F}_{r} \boldsymbol{a}-\boldsymbol{f}_{r}\right)^{T}\left(\boldsymbol{F}_{r} \boldsymbol{a}-\boldsymbol{f}_{r}\right)+\left(\boldsymbol{F}_{i} \boldsymbol{a}-\boldsymbol{f}_{i}\right)^{I}\left(\boldsymbol{F}_{i} \boldsymbol{a}-\boldsymbol{f}_{i}\right) \\
y= & \boldsymbol{a}^{T}\left[\boldsymbol{F}_{r}^{T} \boldsymbol{F}_{r}+\boldsymbol{F}_{i}^{T} \boldsymbol{F}_{i}\right] \boldsymbol{a}-\boldsymbol{a}^{T}\left[\boldsymbol{F}_{r}^{T} \boldsymbol{f}_{r}+\boldsymbol{F}_{i}^{T} \boldsymbol{f}_{i}\right] \\
& -\left[\boldsymbol{f}_{r}^{T} \boldsymbol{F}_{r}+\boldsymbol{f}_{i}^{T} \boldsymbol{F}_{i}\right] \boldsymbol{a}+\boldsymbol{f}_{r}^{T} \boldsymbol{f}_{r}+\boldsymbol{f}_{i}^{T} \boldsymbol{f}_{i}
\end{aligned}
$$

In order to minimize $y$, the gradient of (12) is taken with respect to the amplitude vector $\boldsymbol{a}$ and set equal to the zero vector.

$$
\frac{\partial y}{\partial \boldsymbol{a}}=\mathbf{0}=2\left[\boldsymbol{F}_{r}^{T} \boldsymbol{F}_{r}+\boldsymbol{F}_{i}^{T} \boldsymbol{F}_{i}\right] \boldsymbol{a}-2\left[\boldsymbol{F}_{r}^{T} \boldsymbol{S}_{r}+\boldsymbol{F}_{i}^{T} \boldsymbol{f}_{i}\right]
$$

The result of the minimization of (10) is a real, square set of linear equations which can be solved by using any linear equation solving technique.

$$
\left[\boldsymbol{F}_{r}^{T} \boldsymbol{F}_{r}+\boldsymbol{F}_{i}^{T} \boldsymbol{F}_{i}\right] \boldsymbol{a}=\boldsymbol{F}_{r}^{T} \boldsymbol{S}_{r}+\boldsymbol{F}_{i}^{T} \boldsymbol{f}_{i}
$$

However, in certain cases, the symmetric matrix premultiplying $\boldsymbol{a}$ is extremely ill-conditioned [14], [15]. In order to see when such cases arise let

$$
\boldsymbol{H}=\boldsymbol{F}_{r}^{T} \boldsymbol{F}_{r}+\boldsymbol{F}_{i}^{T} \boldsymbol{F}_{i}
$$

and examine the case of a two reflection problem for simplicity (see (16), shown at the bottom of the page). One obvious case that will cause $\boldsymbol{H}$ to be ill-conditioned occurs when the values of $t_{1}$ and $t_{2}$ are nearly equal. When the values of $t_{1}$ and $t_{2}$ are exactly equal, $\boldsymbol{H}$ becomes singular. In order to deal with this problem, the singular value decomposition technique [14]. $[22]$ is used to solve (14). When any singular value is found to be less than 0.01 , its value is set equal to zero before the final computation of $a$ is performed. This extracts the minimum norm solution to (14), which is precisely what is sought.

The original LS optimization problem (5) has been decomposed into a linear problem whose solution yields the amplitudes $A_{i}$, and a highly nonlinear problem whose solution, it will be shown, yields the delays $t_{i}$. It is important to recognize that the solution of (14) for the amplitudes $A_{i}$ requires that a set of delays $t_{i}$ be utilized in forming the entries of the matrix $\boldsymbol{H}$.

The following optimization procedure may be followed for extracting the $A_{i}, t_{i}$ pairs:

1) Choose the number $N$ of $A_{i}, t_{i}$ pairs to be determined.

2) Assume a sequence of $t_{i}$ values and solve (14) for the optimal $A_{i}$.

3) Search for the global minimum of (5) using the amplitudes found from the solution of (14).

It will be shown in the next section that the geometric interpretation of the LS objective function $J$, subject to the solution of (14), is a multiple minima surface with grooves aligned with the delay axes. The grooves in this surface can

$$
\begin{aligned}
\boldsymbol{F} & =\left[\begin{array}{cccc}
\cos \left(\omega_{1} t_{1}\right)-j \sin \left(\omega_{1} t_{1}\right) & \cos \left(\omega_{1} t_{2}\right)-j \sin \left(\omega_{1} t_{2}\right) & \cdots & \cos \left(\omega_{1} t_{N}\right)-j \sin \left(\omega_{1} t_{N}\right) \\
\cos \left(\omega_{2} t_{1}\right)-j \sin \left(\omega_{2} t_{1}\right) & \cos \left(\omega_{2} t_{2}\right)-j \sin \left(\omega_{2} t_{2}\right) & \cdots & \cos \left(\omega_{2} t_{N}\right)-j \sin \left(\omega_{2} t_{N}\right) \\
\vdots & \vdots & \ddots & \vdots \\
\cos \left(\omega_{n} t_{1}\right)-j \sin \left(\omega_{n} t_{1}\right) & \cos \left(\omega_{n} t_{2}\right)-j \sin \left(\omega_{n} t_{2}\right) & \cdots & \cos \left(\omega_{n} t_{N}\right)-j \sin \left(\omega_{n} t_{N}\right)
\end{array}\right] \\
\boldsymbol{H}^{2 \times 2} & =\left[\begin{array}{ccc}
\sum_{i=1}^{n} \cos ^{2}\left(\omega_{i} t_{1}\right)+\sin ^{2}\left(\omega_{i} t_{1}\right)=n & \sum_{i=1}^{n} \cos \left(\omega_{i} t_{1}\right) \cos \left(\omega_{i} t_{2}\right)+\sin \left(\omega_{i} t_{1}\right) \sin \left(\omega_{i} t_{2}\right) \\
\sum_{i=1}^{n} \cos \left(\omega_{i} t_{1}\right) \cos \left(\omega_{i} t_{2}\right)+\sin \left(\omega_{i} t_{1}\right) \sin \left(\omega_{i} t_{2}\right) & \sum_{i=1}^{n} \cos ^{2}\left(\omega_{i} t_{2}\right)+\sin ^{2}\left(\omega_{1} t_{2}\right)=n
\end{array}\right]
\end{aligned}
$$


be searched sequentially and the global minimum found with little computation. The global minimum corresponds to the set of optimum reflection amplitudes and delays and hence to target range. The next section presents FSCW measurements made using an HP-8510 network analyzer that are analyzed using both the standard IFFT processing technique as well as the proposed optimization approach.

\section{EXPERIMENTAL RESULTS}

This section presents FSCW measurements made using an HP-8510 network analyzer in two different experimental setups. First, measurements of the reflections in an air-line coaxial cable section, the 7-mm Beatty Standard, are presented. Then, measured radar data from a test apparatus is examined. A comparison of the performance of extracting the reflection time delays using the standard IFFT approach, as used by the HP-8510 network analyzer internal computer, and the optimization approach is presented.

Consider the 7-mm Beatty Standard [1], [10], [13] terminated in a matched load and its theoretical bounce diagram as shown in Fig. 1. The Beatty Standard presents two stepdiscontinuities in the characteristic impedance of the line. These discontinuities are produced by an abrupt change in the outer diameter of the inner conductor. The bounce diagram shows the theoretical amplitude and delay of each reflection for measurement calibrated to the input port of the Beatty Standard. Measurements of the reflection coefficient $s_{11}$ were taken over two different bandwidths using an HP-8510 network analyzer. The first set of measurements was taken over the band $45 \mathrm{MHz}-18 \mathrm{GHz}$ using 801 evenly spaced frequencies. The resulting IFFT is shown in Fig. 2. A rectangular window [23] was utilized to maximize the resolution between the reflections. The $18-\mathrm{GHz}$ bandwidth is large enough to clearly distinguish the reflections. In addition, the amplitudes and delays of the first three reflections are very accurate.

The second set of measurements was taken over the band 4-6 $\mathrm{GHz}$ using 801 evenly spaced frequencies, and the resulting IFFT is shown in Fig. 3. The $2-\mathrm{GHz}$ bandwidth is so small that the two reflections ( $83 \mathrm{ps}, 583 \mathrm{ps}$ ) have merged into a single pulse. In this case the IFFT cannot resolve the two reflections. In addition, the location of the peak in the impulse response is biased [24] away from the correct location of either of the two reflections. Hence, when two reflections are so close in time that the IFFT cannot resolve them, accuracy is lost as well.

Consider the objective function (5) for two assumed delays, one scanned from $0-0.8 \mathrm{~ns}$ along one delay dimension and the other from $0.06-0.1 \mathrm{~ns}$ using the Beatty Standard data and 18 $\mathrm{GHz}$ bandwidth. This function may be interpreted as a surface above the "delay" plane as shown in Fig. 4. It is important to recognize that every value of $J\left(t_{i}, t_{j}\right)$ is the result of solving (14) using each delay pair $t_{i}, t_{j}$ in the domain shown. The surface shown in Fig. 4 has a single global minimum that corresponds the optimum delay pair $\left(t_{1}=83 \mathrm{ps}, t_{2}=583\right.$ ps). The grooves in the objective function extend along the delay axes. The deeper groove at $0.083 \mathrm{~ns}$ is due to the larger amplitude reflection caused by the first discontinuity of the
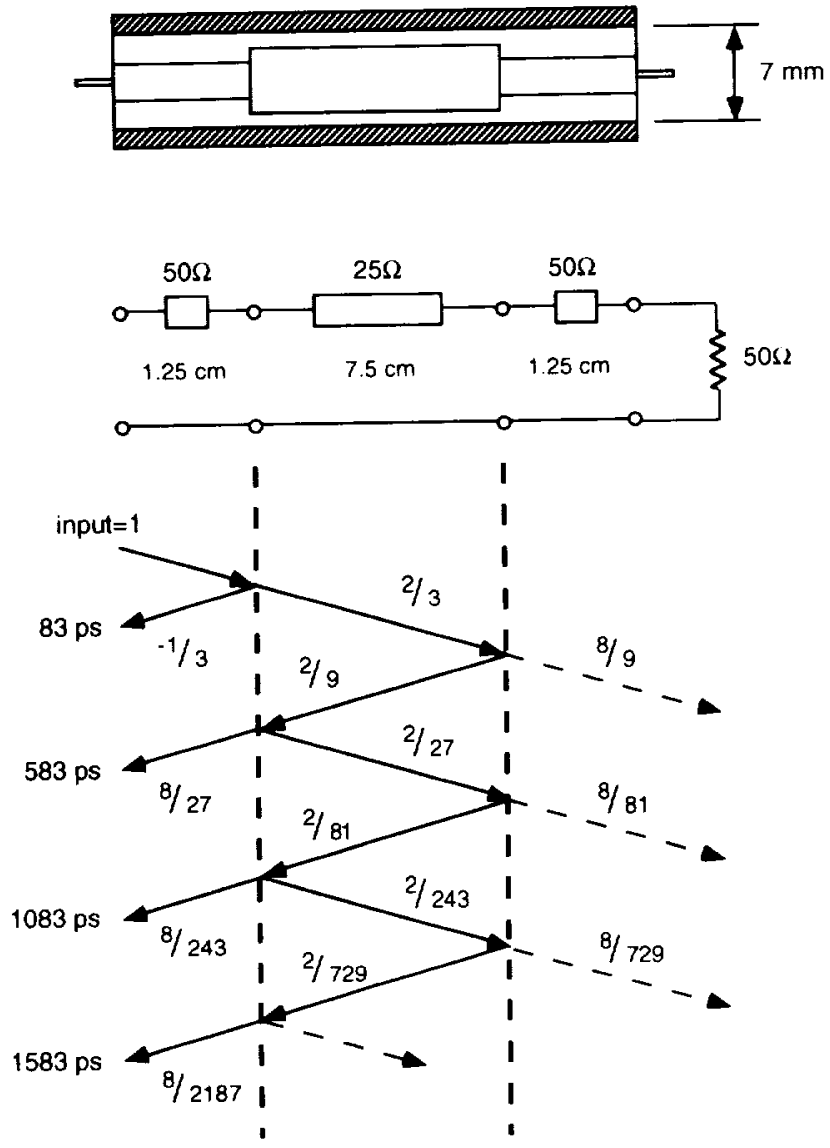

Fig. 1. 7-mm coaxial Beatty Standard terminated in a matched load and the resulting bounce diagram for a unit impulse stimulus.

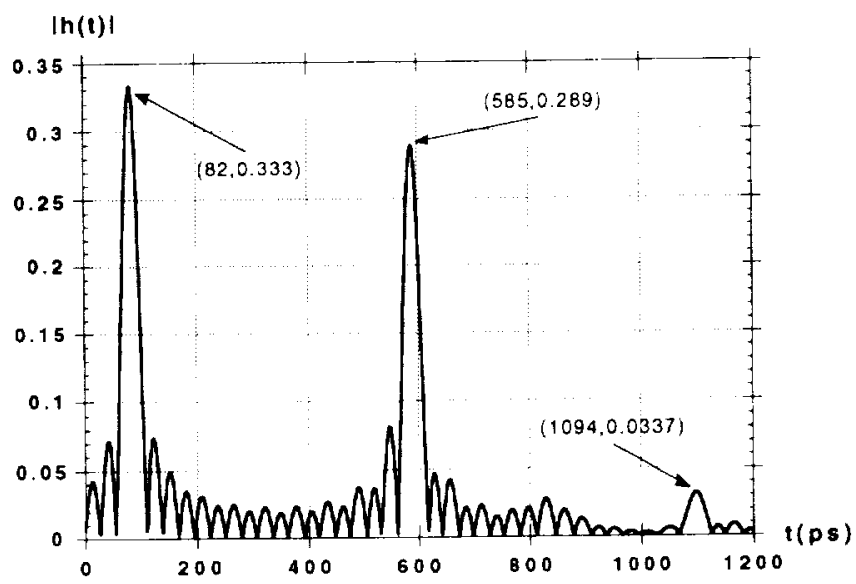

Fig. 2. Inverse FFT of 801 measurements of the reflection coefficient at the input port of the 7-mm Beatty Standard shown in Fig. 1. The bandwidth used in this measurement is $18 \mathrm{GHz}$.

Beatty Standard coaxial line (amplitude $=-0.3333$ ). The groove at $0.583 \mathrm{~ns}$ is due to the slightly smaller amplitude reflection from the second discontinuity (amplitude $=0.297$ ). The surface has multiple local minima, hence a simple descent algorithm will fail, in general, to locate the global minimum. One approach that will be demonstrated here is to use a grid search algorithm on the objective function. Grid search is a method of finding the global minimum by finding the least value of $J$ along one delay dimension while holding the other 


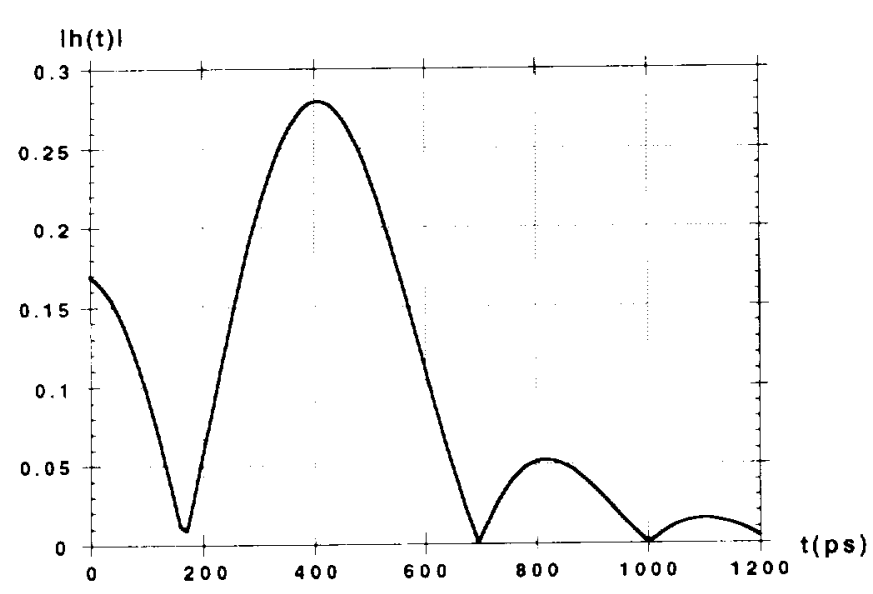

Fig. 3. Inverse FFT of 801 measurements of the reflection coefficient at the input port of the 7-mm Beatty Standard shown in Fig. 1. The bandwidth used in this measurement is $2 \mathrm{GH}$.

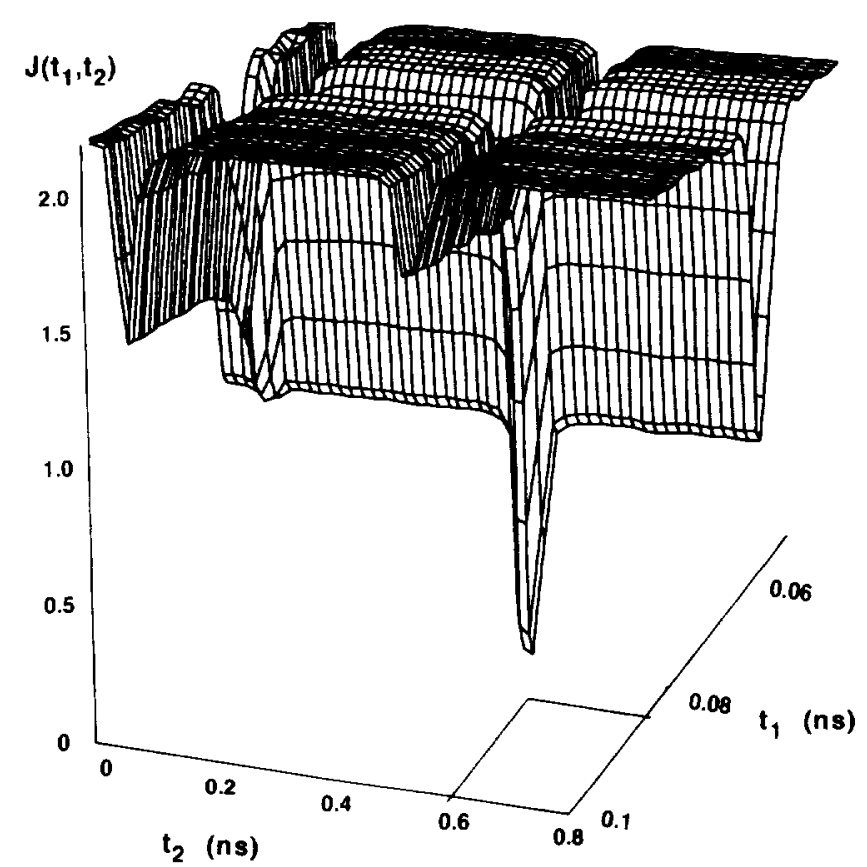

Fig. 4. Amplitude optimized objective function constructed from 801 reflection coefficient measurements of the Beatty Standard (Fig. 1) using a bandwidth of $18 \mathrm{GH} z$.

delay fixed. Then, once the least value of $J$ is found in that dimension, the corresponding delay is held fixed, and the search is performed along the other dimension. By performing multiple iterations in this fashion, the global minimum is found once the delays are no longer updated. The global minimum for the 18-GHz case (Fig. 4) was found in two iterations to be $t_{1}=0.082 \mathrm{~ns}, t_{2}=0.588 \mathrm{~ns}$, very close to both the theoretical delays as well as the estimates produced by the IFFT approach.

The optimal processing technique is globally optimal in the sense that the minimized objective function (5) has many local minima and a search procedure is used to find the absolute minimum. This global minimum determines the best set of reflection amplitudes and delays given the frequency domain data and the model that assumes that the radar channel has distinctly reflective scatterers.

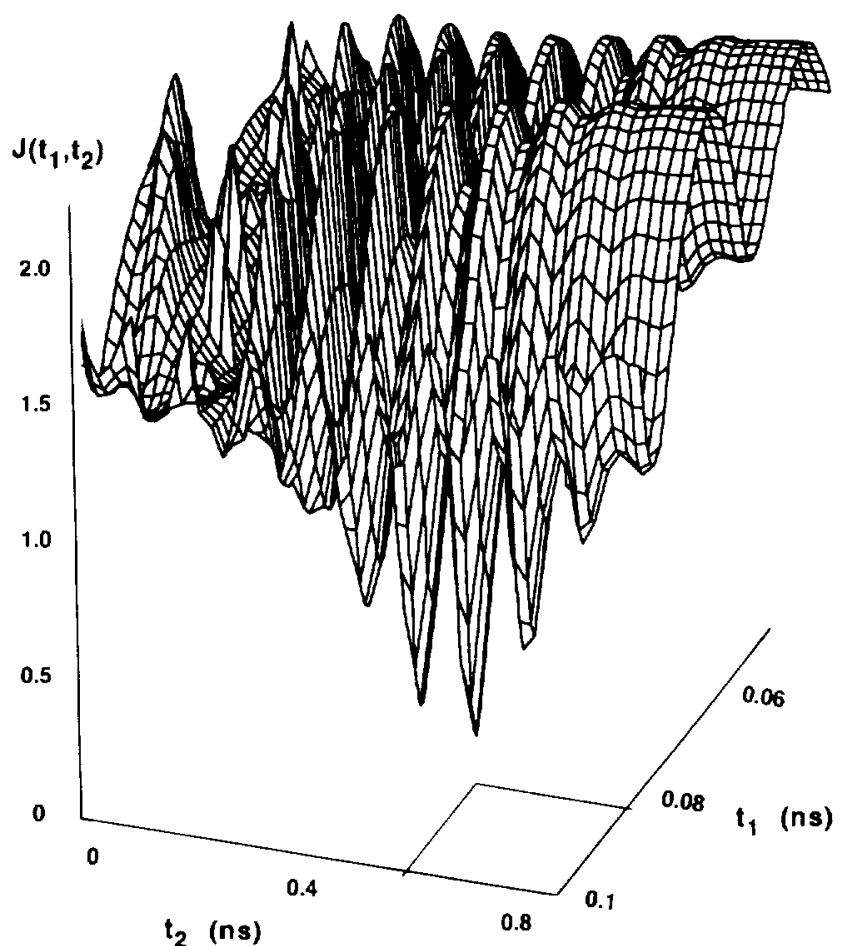

Fig. 5. Amplitude oplimized objective function constructed from $801 \mathrm{rc}$ flection coefficient measurements of the Beatly Standard (Fig. 1) using a bandwidth of $2 \mathrm{GHz}$.

Without a priori knowledge of the number of scatterers, there is no way to know, at least initially, the optimal dimension in which to search for the global minimum. The more interesting case occurs when the bandwidth is reduced to 2 $\mathrm{GHz}$ and the IFFT is useless for determining the range to either of the first two reflections. Applying the optimal signal processing algorithm to the frequency data for this case results in the objective function illustrated in Fig. 5. There still exist grooves in the objective function, each of which is aligned with a delay axis. However, there are several grooves whose minima lie between the values 0.083 and $0.583 \mathrm{~ns}$. There does exist a single global minimum in this domain, and the delay values obtained using two iterations of grid search to find it are 0.082 and $0.587 \mathrm{~ns}$. This result alone shows the resolution enhancement offered by the optimization approach over the standard IFFT as used by the HP-8510 network analyzer.

It is often the case that the number of scatterers is unknown. If the Beatty Standard $18-\mathrm{GHz}$ data is scanned in only one delay dimension using the optimization approach, the objective function shown in Fig. 6 results. Three cases are presented to illustrate the effect of additive white Gaussian noise (zero mean), in addition to the effect of optimizing in a dimension less than the number of scatterers. When no noise is added to the data, the two primary reflections are clearly evident by the minima in the objective function. The locations in time of these minima correspond, with high accuracy, to the two-way travel times to the discontinuities in the Beatty Standard.

By scanning the data in one delay dimension and observing distinct multiple minima in the objective function, it is possible to detect the presence and location of multiple scatters, as well as estimate the "proper" dimension in which to perform a 


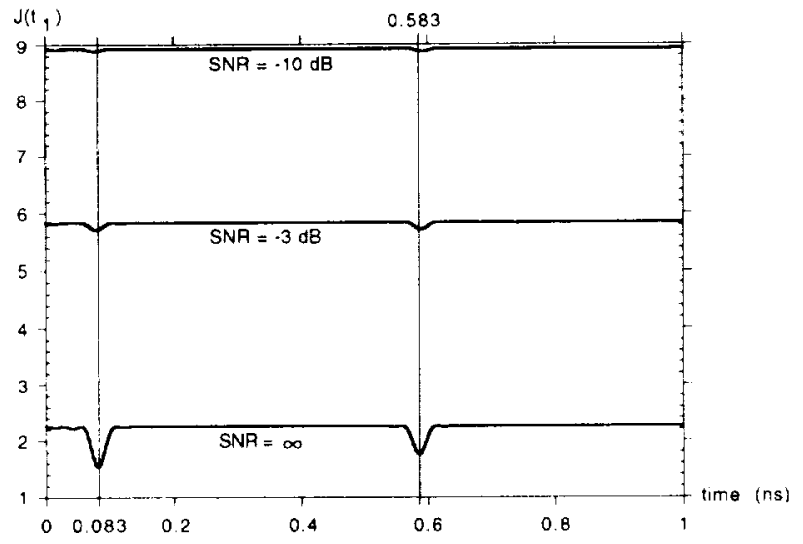

Fig. 6. Effects of noise on the ampltitude optimized objective function in one delay dimension. 801 measurements of the reflection coefficient at the input port of the 7-mm Beatty Standard using a bandwidth of $18 \mathrm{GHz}$.

subsequent optimization. Fig. 6 also illustrates the degradation in the one-dimension objective function as the SNR is reduced. The SNR is defined here to be

$$
\mathrm{SNR}_{\mathrm{d} \mathrm{B}}=10 \log \frac{\sum_{i=1}^{n}\left|H_{M}\left(\omega_{i}\right)\right|^{2}}{\sum_{i=1}^{n} n^{2}\left(\omega_{i}\right)}
$$

where $n\left(\omega_{i}\right)$ is the Gaussian noise sample added to the measured reflection coefficient $H_{M}\left(\omega_{i}\right)^{1}$ at frequency $\omega_{i}$. As the noise level is increased, the mean value of the objective function increases due to the power contributed by the noise. This trend is evidenced by the three plots shown in Fig. 6 . The upward shift in the objective function as the SNR is reduced does not degrade the detectability of the scatterers. However, as the noise level is increased, the depth of the minima decreases, and hence confidence of detection is reduced. At an SNR of $-10 \mathrm{~dB}$, the two discontinuities are still detectable, and the locations in time of the objective function minima still provide an accurate estimate of their location. Fig. 7 presents a comparison of the same three objective functions of Fig. 6 with each function shifted to a common maximum value. This figure illustrates the relative depths of the minima as a function of noise level for a single noise realization. As the noise level is increased, the ratio of the minimum value to the first side-lobe amplitude diminishes, and hence the confidence of detection diminishes as well. When the noise power is increased from zero to ten times the signal power, the depth of the primary minima is reduced by approximately an order of magnitude. The optimized amplitude as a function of delay for the three cases of Figs. 6 and 7 is shown in Fig. 8. The amplitude of the perturbations in the optimization process are more clearly defined in this figure. The perturbation amplitude clearly increases with the noise level, and the deviation of the estimated amplitudes from the correct values is biased toward an overestimate. It remains the subject of future research to quantify the statistical characteristics of the optimization

\footnotetext{
In the case of an actual noisy measurement. $H_{: 11}\left(\omega_{i}\right)$ contains the noise.
}

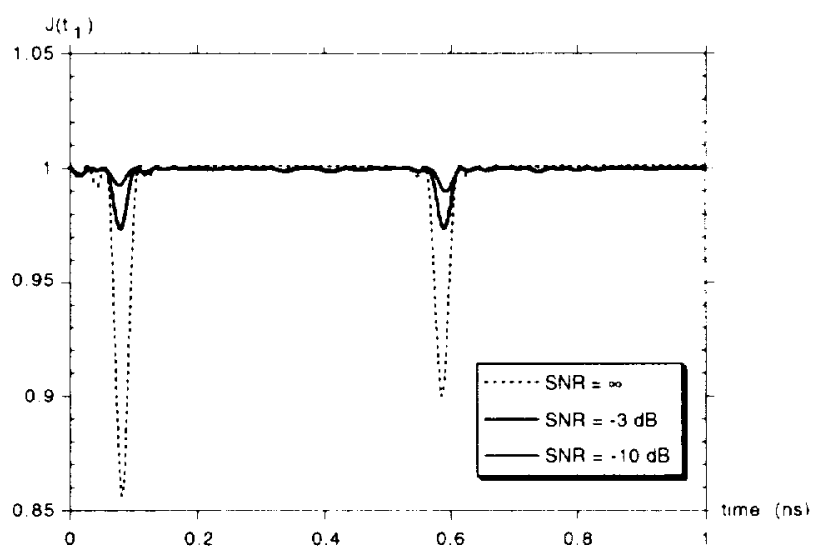

Fig. 7. Effects of noise on the ampltitude optimized objective function in one delay dimension. 801 measurements of the reflection coefficient at the input port of the 7-mm Beatty Standard using a bandwidth of $18 \mathrm{GHz}$. The objective functions have been shifted to a common maximum value.

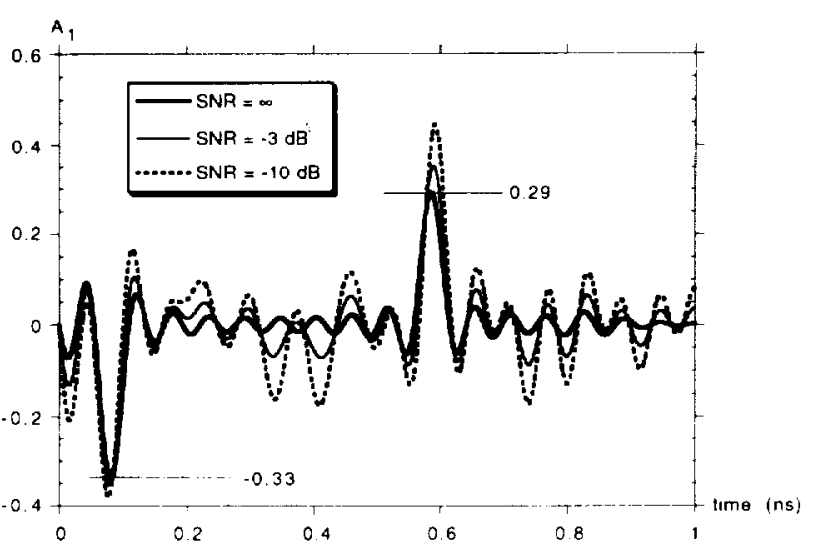

Fig. 8. Effects of noise on the optimal amplitude of a single impulse as a function of time delay. 801 measurements of the reflection coefficient at the inpul port of the 7-mm Beatty Standard using a bandwidth of $18 \mathrm{GHz}$.

process as a function of noise, as well as bandwidth and target range.

In order to test the applicability of the optimization approach to detecting the range to a highly reflective target in the presence of an interfering reflection using FSCW radar data, a test fixture was constructed at NASA LaRC. This fixture is shown in Fig. 9 along with its physical connection to the network analyzer via a rectangular-to-circular waveguide transition. The network analyzer is calibrated to the input port of the waveguide as shown in the diagram. The dielectric interferer is composed of a 0.25 -in.-thick layer of silicon dioxide, or quartz glass $\left(\epsilon_{r}=3.78\right)$, and a 0.5 -in.-thick layer of polyurethane foam $\left(\epsilon_{r}=1.4\right)$. A 0.5 -in.-thick aluminum plate is used for the aperture ground plane. The target is formed by the presence of another 0.5 -in.-thick aluminum plate parallel to the aperture plane of the circular horn antenna. Target range is set by moving the mobile target.

In the first set of measurements using the test apparatus, the target was set at a distance of 12 in. from the outer surface of the quartz glass. The two primary reflections will be due to the high dielectric constant quartz glass and the metal plate target. 801 measurements of the reflection coefficient 


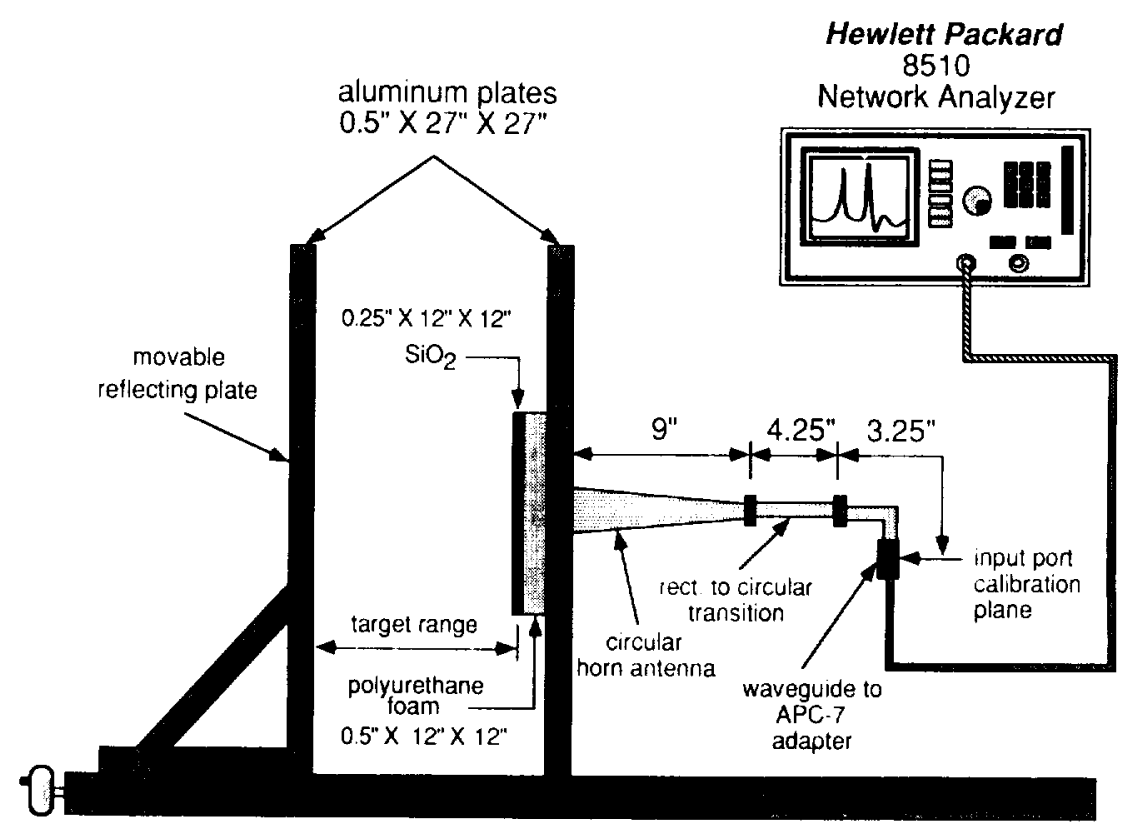

Fïg. 9. Test apparatus.

were made from 14-18 GHz, spanning a total bandwidth of 4 GHz. The IFFT of the measured radar data is shown in Fig. 10. The first three reflections are clearly resolved. The time delay corresponding to the first reflection, due to the quartz glass interferer, is difficult to calculate accurately because the circular horn antenna as well as the parallel plate waveguide formed by the two aluminum plates stimulate the creation of several TE and TM modes. However, the prediction of the time delay to the interfering reflection from the quartz glass is not the primary interest here. The primary concern is target range resolution and accuracy. Detecting accurately the time delay from the reflection due to the quartz glass to the metal plate target is the objective. The IFFT shown in Fig. 10 indicales there is a delay of $1.97 \mathrm{~ns}$ from the quartz glass to the metal plate. Using the free space velocity of propagation, the twoway distance of $24 \mathrm{in}$. in air requires $2.032 \mathrm{~ns}$. Since each reflection from the quartz glass is assumed to be a single impulse, two unresolved impulses resulting from the front and back surfaces, an additional delay of $41 \mathrm{ps}$ is included to account for the $0.25 \mathrm{in}$. of travel through the glass. Thus, the target range is defined to be the distance from the center of the glass to the metal plate. The total theoretical two-way travel time is $2.073 \mathrm{~ns}$. A time delay estimate of $1.97 \mathrm{~ns}$ is produced by the IFFT. Thus, the error in the time delay estimate produced by the IFFT using $4 \mathrm{GHz}$ bandwidth is 103 ps and corresponds to a range estimate error of approximately 0.608 in. $(1.545 \mathrm{~cm})$.

The objective function surface for 801 measurements over a 4-GHz bandwidth is shown in Fig. 11. This surface clearly shows grooves along the delay axes. The domain of this plot was chosen to display the symmetry of the objective surface. As shown, there are two global minima one with $t_{1}=3.47$ $\mathrm{ns}, t_{2}=5.43, \mathrm{~ns}$ and the other with $t_{1}=5.43 \mathrm{~ns}, t_{2}=3.47$ $\mathrm{ns}$, which are exactly the same solution to the problem. The deeper groove at $3.47 \mathrm{~ns}$ is due to the reflection from the quarts.

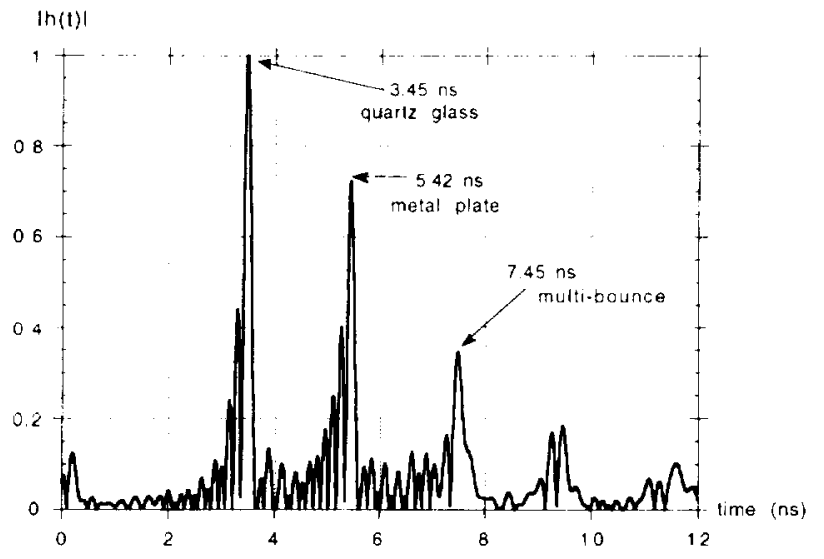

Fig. 10. Nurmalized Inverse FFT of 801 measurements of the reflection coefficient at the input port of the test apparatus shown in Fig. 9. The bandwidth used in this measurement is $4 \mathrm{GHz}$, and the target range is 12 in.

glass and the other groove is due to the weaker target reflection at $5.43 \mathrm{~ns}$. Between the two major grooves lie several small amplitude grooves, also aligned with the delay axes. The top of the surface is relatively smooth and is characteristic of a "wellbehaved" objective function. If the number of measurements is reduced from 801 to 21 while holding the bandwidth and target range constant at $4 \mathrm{GHz}$ and 12 in., respectively, the objective function surface that results is shown in Fig. 12. Although the top of the surface is not as smooth as using 801 measurements, the small amplitude perturbations do not increase the number of grid search iterations required to find either of the global minima shown. The two global minima are deep and narrow enough to clearly indicate the presence of two significant reflections. This result shows the relative immunity of the optimization approach to reducing the size of the data set.

In the next set of measurements, the target was initially set at a distance of $12 \mathrm{in}$. from the outer surface of the quartz 


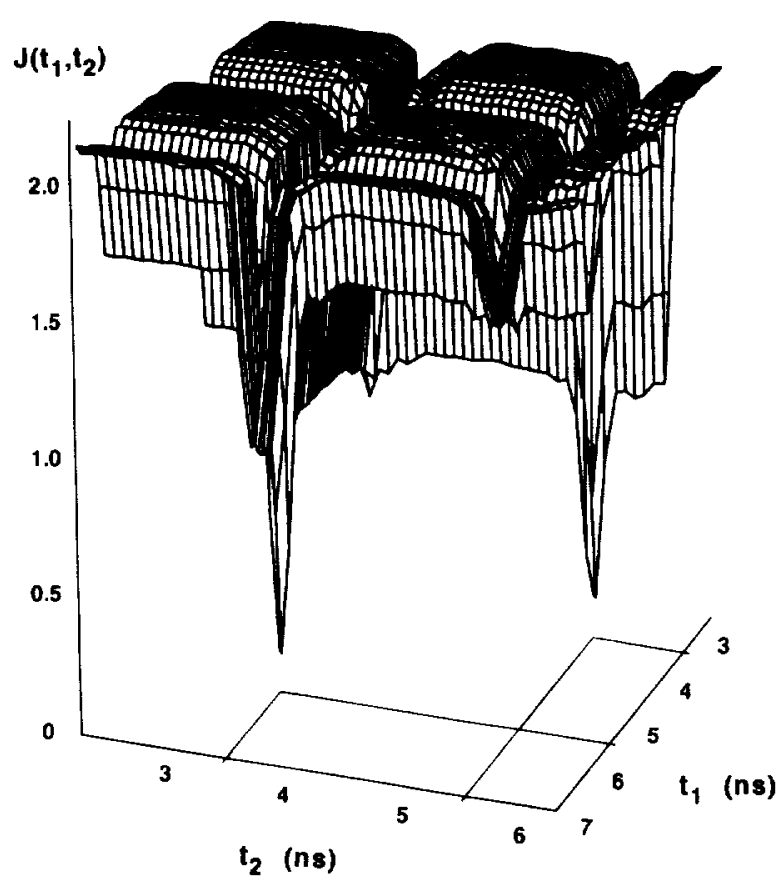

Fig. 11. Amplitude optimized objective function constructed from 801 reflection coefficient measurements of the test apparatus (Fig. 9) using a bandwidth of $4 \mathrm{GHz}$. Target is at a distance of 12 in. from the outer surface of the quartz glass.

glass. One-hundred and one measurements of the reflection coefficient were made from $14-14.5 \mathrm{GHz}$, spanning a total bandwidth of only $500 \mathrm{MHz}$. The IFFT of the measured radar data is shown in Fig. 13. The bandwidth was selected such that the IFFT has a resolution marginally able to detect the presence and correct location of the first two reflections. When the target distance is reduced to 2.25 in. from the outer surface of the quartz glass, the resulting IFFT is shown in Fig. 14. The narrow bandwidth spanned by the measurement sequence is so small that several reflections have merged into a single lobe of the IFFT. The 2.25 in. distance from the outer surface of the quartz glass to the metal plate target corresponds to $0.381 \mathrm{~ns}$ of two-way travel time. Including $41 \mathrm{ps}$ of delay within the quartz glass results in a total theoretical two-way travel time of $0.422 \mathrm{~ns}$. Since the metal plate target is so close to the interferer, there are many reflections between the quartz glass and the aluminum plate. These reflections are separated by $0.422 \mathrm{~ns}$ in time. The main lobe in the IFFT spans approximately 2 ns, enough time to "swallow" nearly 3 of these multi-bounce reflections. The time delay corresponding to the second major peak in the IFFT of Fig. 14 is $4.8 \mathrm{~ns}$ and has no value in assessing the range to the target. This is the best performance the IFFT has to offer for determining the target range under the bandwidth constraint of $500 \mathrm{MHz}$ and 101 frequency measurements.

The objective function surface resulting from the data set whose parameters are $4 \mathrm{GHz}, 101$ measurements, and target distance 12 in. from the quartz glass is shown in Fig. 15. The domain scanned has been chosen once again to display the symmetry of the objective function surface. The two global minima each provide reflection delay estimates of $3.47 \mathrm{~ns}$ and $5.50 \mathrm{~ns}$, resulting in a target delay estimation error of only 43

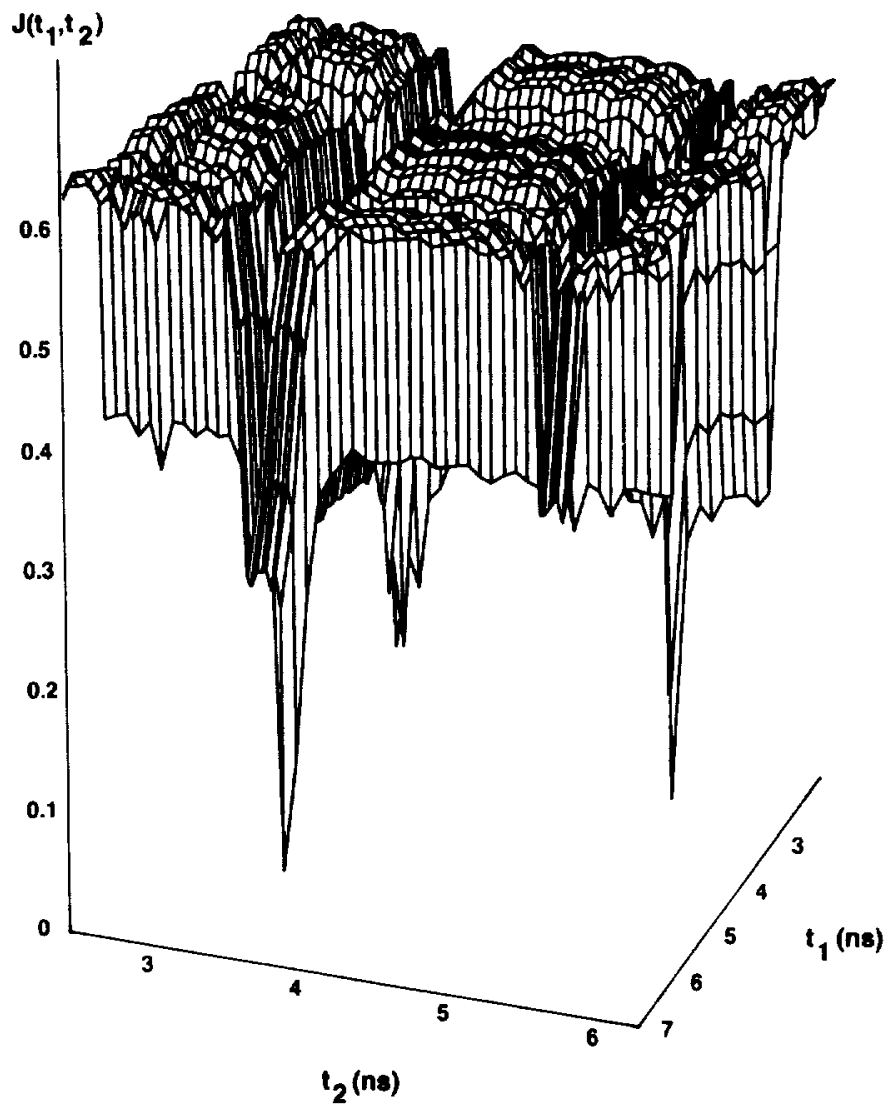

Fig. 12. Amplitude optimized objective function constructed from 21 reflection coefficient measurements of the test fixture (Fig. 9) using a bandwidth of $4 \mathrm{GHz}$. Target is at a distance of $12 \mathrm{in}$. from the outer surface of the quartz glass.

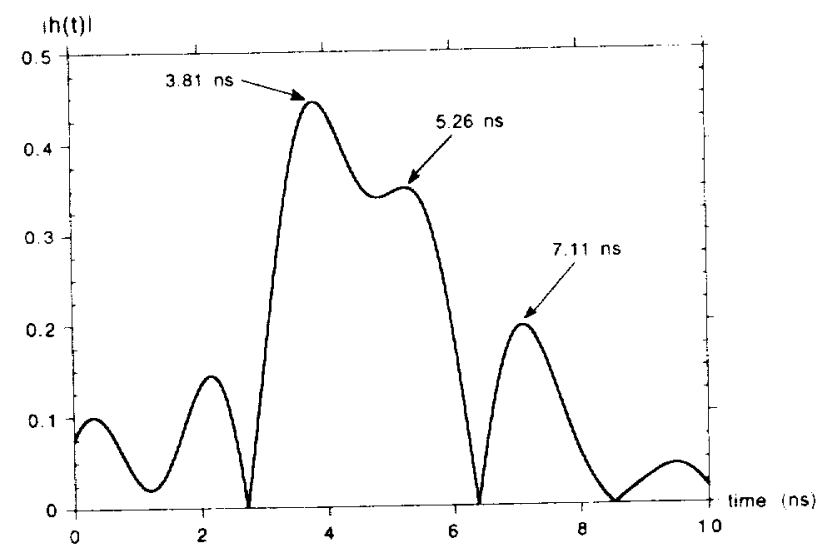

Fig. 13. Normalized Inverse FFT of 101 measurements of the reflection coefficient at the input port of the test apparatus shown in Fig. 9. The bandwidth used in this measurement is $500 \mathrm{MHz}$. The Target is at a distance of $12 \mathrm{in.} \mathrm{from} \mathrm{the} \mathrm{outer} \mathrm{surface} \mathrm{of} \mathrm{the} \mathrm{quartz} \mathrm{glass.}$

ps. If the edge of the surface defined by $t_{i}=8 \mathrm{~ns}$ is examined closely, three distinct troughs in the surface can be observed. The first trough is located at a delay value of $3.47 \mathrm{~ns}$ and corresponds to the strongest reflection that is due to the quartz glass. The second trough is located at a delay value of $5.50 \mathrm{~ns}$ and is due to the metal plate target. The third trough is located at a delay value of $7.51 \mathrm{~ns}$ and is due to the first multi-bounce reflection between the metal plate and quartz glass. Hence, although the surface presented is the result of minimizing a 


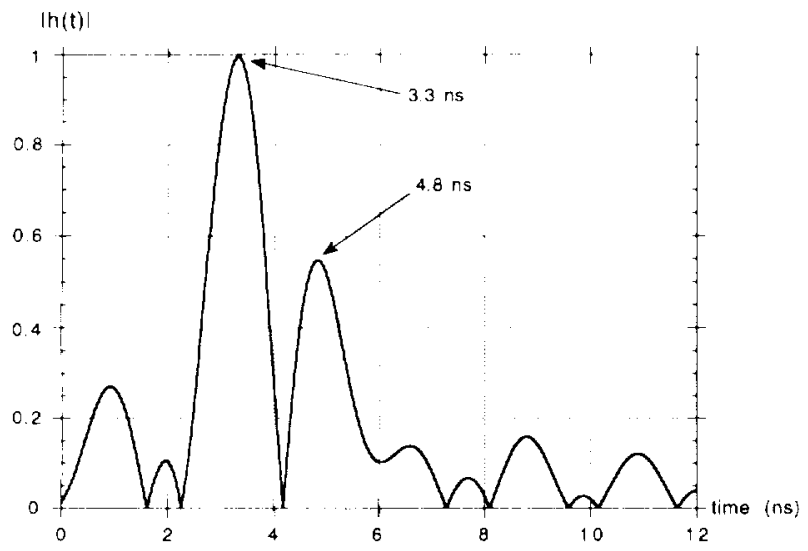

Fig. 14. Normalized Inverse FFT of 101 measurements of the reflection coefficient at the inpul port of the test apparatus shown in fig. 9. The bandwidth used in this measurement is $500 \mathrm{MH} / \mathrm{s}$, and the larget range is 2.25 in.

two-delay objective, the surface contains information enabling the location of higher-order reflections. If the target is moved to a distance of 2.25 in. from the quartz glass while holding the bandwidth and number of measurements constant at $500 \mathrm{MHz}$ and 101 , respectively, the objective function surface shown in Fig. 16 results. This objective function surface may appear to lack structure. However, the surface has grooves running along the delay axes that can be scanned successfully using the grid search algorithm. Evidence of these grooves may be seen by carefully examining the right half of the surface. The ridges in the surface indicate the presence of the grooves. The surface also possesses a macroscopic bowl shape, albeit very "noisy," in the domain shown. There are two global minima, either of which produces the delay estimates $t_{1}=3.53 \mathrm{~ns}$, $t_{2}=3.95 \mathrm{~ns}$. These delay values were found by polishing the result of a first-pass grid search with an additional grid search. In the first pass, the domain scanned was $1-6 \mathrm{~ns}$ in each delay dimension using $50 \mathrm{ps}$ increments. After 16 iterations of grid search, the resulting time delays are $t_{1}=3.55 \mathrm{~ns}$, $t_{2}=3.95 \mathrm{~ns}$. Then, a second pass was performed over the domain 3.5-3.6 ns in the first delay dimension and 3.9-4.0 ns in the second delay dimension using $1 \mathrm{ps}$ increments. After 12 itcrations of grid search in the second pass, the resulting time delays are $t_{1}=3.53 \mathrm{~ns}, t_{2}=3.95 \mathrm{~ns}$. The impulse response produced by the optimization technique is shown in Fig. 17. The most important measurement is the difference in time delay between the two reflections. This delay difference is found to be $t_{2}-t_{1}=0.42 \mathrm{~ns}$. The theoretical value was calculated to be $0.422 \mathrm{~ns}$. Hence, a delay error of only $2 \mathrm{ps}$ is produced by the optimization approach in this case. This 2-ps error in the delay estimate corresponds to approximately 0.3 $\mathrm{mm}$. This result clearly shows the resolution capability of the optimization approach. The IFFT for this measurement case (Fig. 14) was totally useless for determining the range to any reflection while the optimization approach has distinctly and accurately resolved both the reflection from the metal plate target as well as the reflection from the quartz glass.

The results for the IFFT and optimization approaches for both the Beatty Standard and test apparatus experiments are presented in Table I.

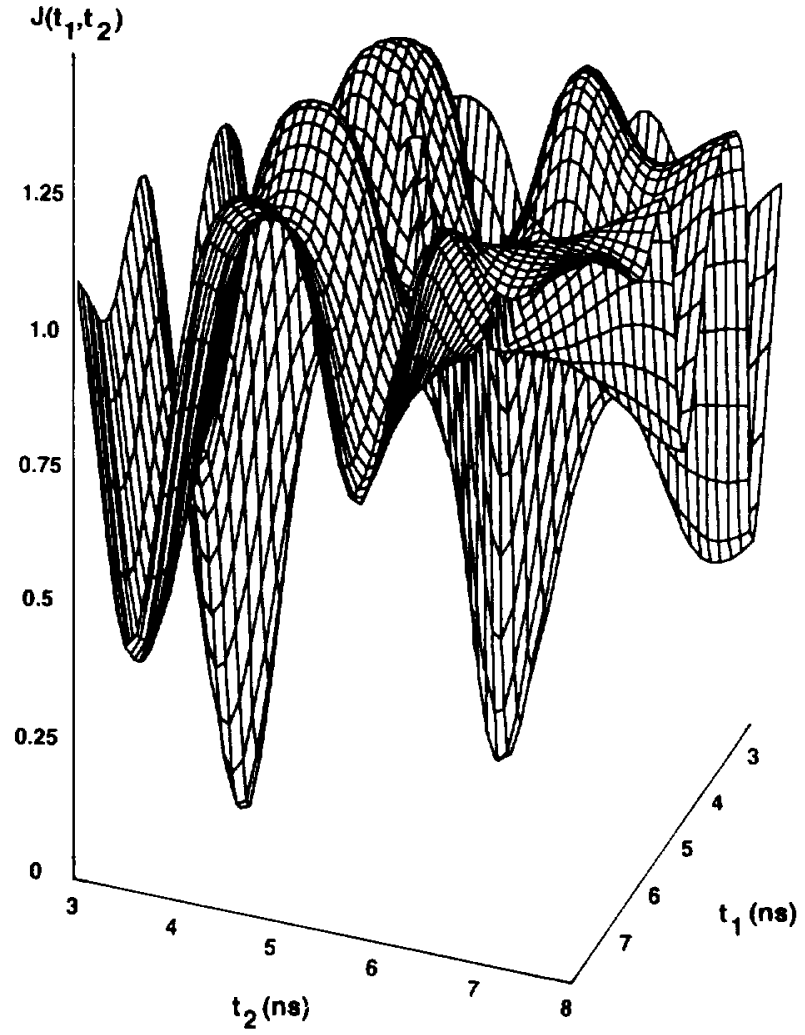

Fig. 15. Amplitude optimized objective function constructed from 101 reflection coefficient measurements of the test apparatus (Fig. 9) using a bandwidth of $500 \mathrm{MHz}$. Tatrget is at a distance of 12 in. from the outer surface of the quartz glass.

\section{CONCLUSION}

This paper has presented the derivation and demonstration of an algorithm for optimal processing of radar data produced by any FSCW system. The derivation is based on minimizing the two-norm of the difference between the sequence of measured reflection coefficients and those produced by a model that assumes the echoes from the radar channel are impulses in the time domain. The algorithm does not require a constant interval between the measurement samples. A performance comparison between the IFFT and optimal processing techniques was presented in the context of two physical measurement sets. In the first set, the Beatty standard was terminated in a matched load and driven by an HP-8510 network analyzer. The measurement bandwidth was reduced and it was shown that while the IFFT produced meaningless reflection range estimates the optimal processing approach was able to clearly resolve the reflections with high accuracy. In the second set of measurements, a test apparatus also driven by an HP-8510 network analyzer was used to illustrate the effects of reducing the bandwidth, number of measurements, and target range. It was shown that when the number of measurements was reduced to 21 , the objective function surface developed a high-frequency, low-amplitude perturbation. The surface global minima, however, were just as sharp and deep as using 801 measurements. This displayed the relative immunity of the optimization approach to reducing the size of the data set. When the target range was reduced from 12.125-2.375 
TABLE I

PERformance COMPaRISON OF THE IFFT AND OPTIMATION APPROACHES FOR the Beatty Standjard and Test Apparatls Experiments. The Target

Range in the Test Apparatls EXPERIMENTS is Defined to be the Distance from the Center of the Quartz Glass to the Metal Plate Target

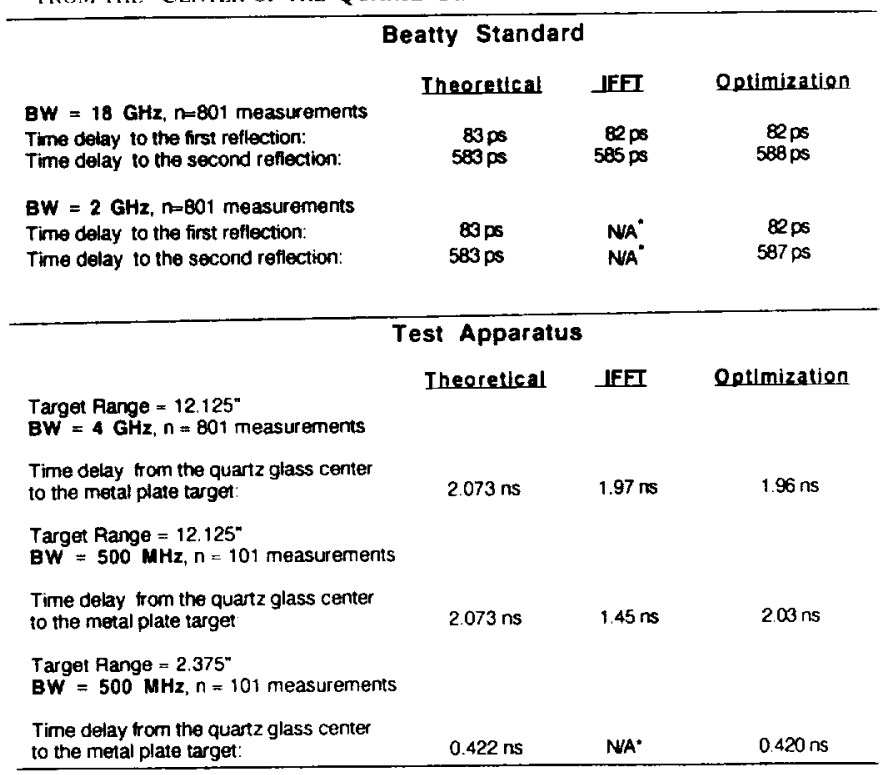

*It is not clear from the IFFT results that there are two reflections. Even if two reflections are assumed, the time delays to the peaks in the IFFT results are erroneous to the point of being meaningless.

in. while holding the bandwidth constant $(500 \mathrm{MHz})$, the range resolution and accuracy deterioration produced by IFFT processing was clearly evident. In this case, the IFFT could not distinguish the presence of two reflections nor could it determine accurately the range to either the interfering reflection or the metal plate target. However, the optimal processing approach could not only resolve the two reflections. but estimate both of their ranges with high accuracy. This result demonstrated the resolution enhancement offered by the optimal processing approach over IFFT processing.

It remains to develop the optimization approach in higher dimensions. The simple grid search algorithm may be inefficient for extracting global minima in higher dimensions, and a more sophisticated search method may be required [25]. One distinguishing characteristic of the optimization approach is its geometrical interpretation which is locating the absolute minimum of a surface lying above a delay plane. By varying measurement parameters and viewing the objective function surface, the effect on the solution can be understood intuitively through visual inspection. A potential advantage of the optimization approach is its ability to incorporate constraints such as time-gating, a technique often implemented in conventional pulsed radar [16]-[21]. Timegating is accomplished by searching only over certain regions in the delay plane.

Future research will be directed toward quantifying the amplitude and frequency of the perturbations that occur in the objective function surface as the bandwidth, or the number of measurements, is reduced. The effects of added noise on the estimated amplitude and delay parameters will be quantified

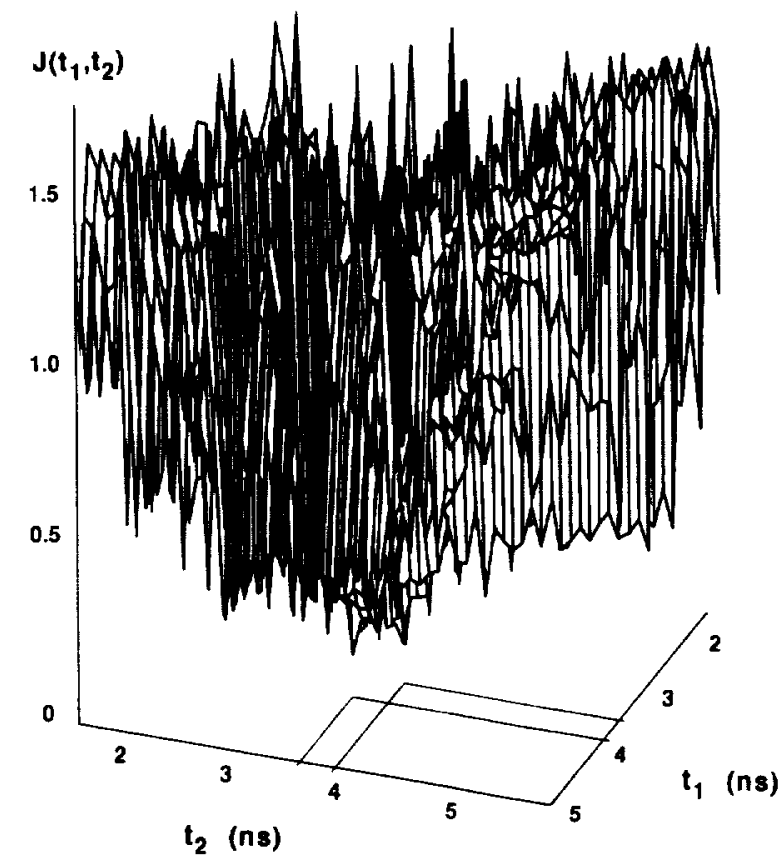

Fig. 16. Amplitude optimized objective function constructed from 101 reflection coefficient measurements of the test apparatus (Fig. 9) using a bandwidth of $500 \mathrm{MHz}$. Target is at a distance of $2.25 \mathrm{in}$. from the outer surface of the quartz glass.

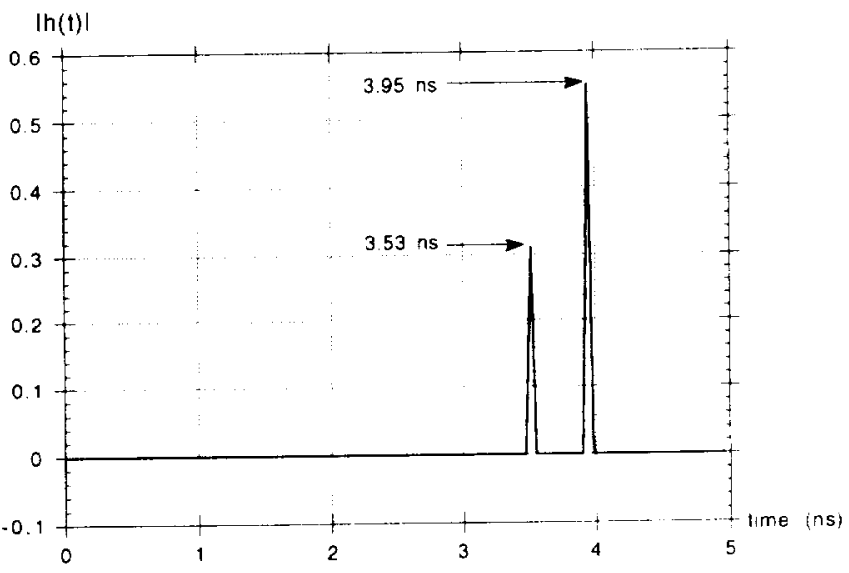

Fig. 17. Normalized Impulse response of the test apparatus of Fig. 6 obtained using grid search ( 16 iterations, 14 iterations polished) on the amplitude-optimized objective function resulting from 101 measurements over $500-\mathrm{MHz}$ bandwidth. The target is at a distance of $2.25^{\prime \prime}$ from the outer surface of the quartz glass.

statistically, along with the confidence of target detection. The benefit of irregular sampling in the frequency domain will be investigated. The number of delay dimensions will be increased and other search procedures investigated for efficient global minimum scanning.

\section{ACKNOWLEDGMENT}

The authors wish to express their thanks to $G$. Monahan and A. Morris at N.C. State University Microwave Labs for providing their expertise with the HP-8510 network analyzer and P. C. Chapman for insightful suggestions. The authors 
are also grateful to the reviewers for providing several valid criticisms of the paper as well as detailed suggestions for its improvement.

\section{REFERENCES}

(1) HP 8510 Nemork Analvaer Operating and Programming Manual. Sanla Rosa, CA: Hewlett-Packard Co., 1984.

(2) G. A Ybarra, S. H. Ardalan, C. P. Hearn, R. E. Marshall, and R. T. Neece, "Detection of target distance in the presence of an interfering reflection using a double side-band suppressed carrier microwave radar system." IEEE Trans. Microwave Theon Tech., May, 1991.

[3] G. A. Ybalra, "High resolution target range estimation in inhomegeneous media using millimeterwave radar," Ph.D. dissertation. 1992.

[4] B. Edde, Radar: Principles. Techonology, Applications. Englewood Cliffs. NJ: Prentice-Hall, 1993.

[5] S. V. Bearse, "European conference showcases innovative commercial applications." Micrentares, Sept. 1977.

[6] T. A. Okrasinski et al., "Microwave interference detection of subsurface water." J. Geotechmical Engines ring Division. Proc: American Society of Civil Enginears, vol. 104, no. GTI, Jan. 1978.

17) R. Jacobson. B. Schiek, and W. Schilz. "Microwave distance meter with a $2.5 \mathrm{~mm}$ resolution." in Pros. 5 th European Microwave Conf., Hamburg. 1975.

$|8|$ D. T. Gjessing. J. Hjelmstad, and Terje Lund. "A multifrequency adaptive radar for detection and identification of objects: Results on preliminary experiments on aircraft against a sea-clutter background," IEEE Trans. Antenn. Propagat., vol. 30, no. 3, May 1982.

[9] H. Vanhamme, "High resolution frequency-domain reflectometry," IEEE. Trans. Instrue Meas. vol. 39, n. 2, Apr. 1990.

[10) Z. A. Maricevic et al.. "Time-domain measurements with the hewlettpackard network analyzer HP-851() using the malrix pencil method," It.EF Trans. Micronueve Theory Tech, vol. 39, no. 3, Mar. 1991.

[II] Y. Hua and T. K. Sarkar. "Matrix pencil method for estimating parameters of exponentially damped/undamped sinusoids in noise." IEFE. Truns. Acoustic Splecech and Signal Processing, vol. 38. no. 5. May 1990.

112] R. Carriere and R. I. Moses, "High resolution radar target modeling using a modified prony estimator." IEEE Trams. Amenn. Propagat. vol. 40, no. I, Jin. 1992

113] R. W. Beatty, "Calculated and measured S11, S21, and group delay for simple types of coaxial and rectangular waveguide 2-port standards," National Bureau of Standards Tech. Note 657, U.S. Department of Commerce, Dec. 1474

$|1+|$ G. H. Golub and C. F. Van L.oan. Matrix Computations. Baltimore, MD: Johns Hopkins University Press. 1989.

[15] C. L. Lawson and R. J. Hanson, Solving Least Squares Problems. Englewood Cliffs, NJ: Prentice-Hall. 1974

[16] Ter'hniques of Radar Reflectivity Medasurement. Norwood. MA: Artech House, 1984

117] Principles and Applications of Millimeterwave Radar. Norwood, MA Artech House, 1987

|18| G. J. Bird. Radar Precision and Resolution. New York: Wiley, 1974.

[19] D. P. Meyer. Radar Target Detection. New York: Academic Press. 1973.

[20] M. 1. Skolnik, Radar Handbook. New York: McGraw Hill Book Company, 1970.

121] D. R. Wehner, High Resolution Radar. Norwood, MA: Artech House, 1987

122] W. H. Press at al. Numerical Recipes in C. New York: Cambridge University Press, 1988.

12.3 F. J. Harris, "On the use of windows for harmonic analysis with the discrete fourier transform," Pros. $I E E E$, vol. 66, Jan. 1978.

124| S. K. Kay, Modern Spectral Estimation: Theory and Application. Fnglewood Cliffs, NJ: Prentice-Hall, 1988

1251 P. R. Bevington, Data Reduction and Error Analusis for the Phvsical Sciences. New York: McGraw-Hill, 1969.

126| G. J. Bird, Radar Precrision and Resolution. New York: John Wiley \& Sons, Inc.

[27] R. B. Collin. Foundations of Microwave Engineering. New York: McGraw Hill, 1966.

281 Collins Publishers. Moderm Radar Ter/mology: London. 1987.

$29 \mid$ R. T. Compton. Adaptive Antennas: Concepts and Performance. Englewood Cliffs, NJ: Prentice-Hall. 1988

130) Adrances in Radar Techmolog: London: IEEE Press, 1985

131) S. M. Kily and S. L. Marple, "Spectrum analysis-A modern perspective," Proc. IFEF, vol. 69. Nov, 1981.
1.32 S. L. Marple, Digital Spectral Analwis with Applications. Englewond Cliffs, NJ: Prentice-Hall, 1987.

133] E. K. Miller. Time Domain Measurements in Electromagnetics. New York: Van Nostrand Reinhold. 1986.

134| B. Noble and J. W. Daniel, Applied Line'ar Algebra. Englewood Cliffs. NJ: Prentice-Hall, 1977

[35] D. A. Noon and M. E. Bialkowski, "An inexpensive microwave distance measuring system," Microwate and Optical Technol. Lett. vol. 6. no. 5. Apr. 1993.

|36| G. Ybarra ef al., "High resolution target range estimation using frequency-stepped CW radar," Micromate Theor Tech. Simp. Dig. Atlantia. GA, 1993

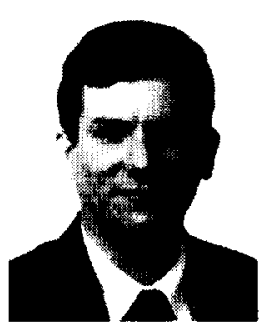

Gary A. Ybarra ( $\mathrm{S}^{\circ} 83-\mathrm{M}^{\circ} \mathrm{9} 2$ ) was born in Hampton, VA in 1960. He received the B.S., M.S. and Ph.D. degrees in electrical enginecring from North Carolina State University, Raleigh, in 1982, 1986 and 1992, respectively.

In 1984 he joined the Center for Communications and Signal Processing (CCSP) at NCSU as a research assistant and investigated properties of multiple impulse speech coding and enhancement algorithms. Continuing his work with CCSP in 1986, he explored the effects of ill-conditioned data on RLS adaptive filters. In 1990). his interest in applying signal processing techniques to microwave and millimeterwave systems led to his current work in radar signal processing in conjunction with the Antennas and Microwave Research Branch at NASA Langley Research Center. In January, 1993 he joined the faculty at Duke University Department of Electrical Engineering where he is an assistant research professor. His research interests include optimal radar signal processing, adaptive signal processing. digital communications, and wireless communication systems.

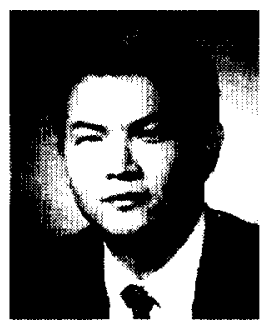

Shawkang M. Wu (S' 9.3) received the M.S. degree in 1988 and is currently working toward the Ph.D. degree, both in electrical engincering at North Carolina State Universily at Rakeigh.

His research interests include digital communicitions, adaptive signal processing, and development of radar signal processing algorithms for uptimal target range estimation.

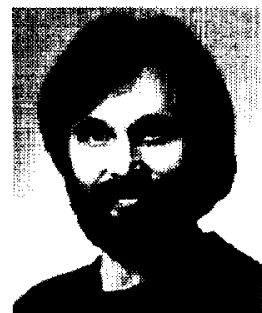

Griff L. Bilbro received the B.S. degree in 1973 from Case Western Reserve University in Cleveland Ohio, and the Ph.D. degree in 1977 from the University of Illinois at Urbana-Champaign, where he was a National Science Foundation Graduate Fellow in Physics.

Until 1984, he designed computer models of complex systems in industry. He joined the Department of Electrical and Computer Engineering at North Carolinal State University where he is now an associate professor

$\mathrm{He}$ is a member of the Center for Communications and Signal Processing as well as the High Frequency Laboralory at NCSUl, where he develops practica engineering techniques tor global oplimization in modeling. device physics. design centering. and image processing. 


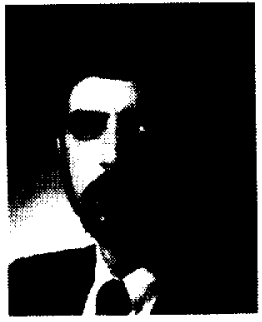

Sasan H. Ardalan ( $\left.\mathrm{S}^{\prime} 75-\mathrm{M}^{\prime} 85\right)$ was born in Tehran, Iran on March 20, 1956. He received the B.Sc., M.Sc., and Ph.D. degrees in electrical and computer engineering from North Carolina State University, Raleigh, in 1977, 1979, and 1983. respectively.

In August 1983, he joined the Center for Communications and Signal Processing (CCSP) at NC State University as a research engineer. In 1985 be became an assistant professor at NC State and then associate professor in 1992 . He was the Principal Investigator of the Physical Layer in the Communications and Networking area in CCSP and developed research programs in computeraided design of advanced digital communication systems including real-time performance evaluation using multi-processor architectures. His research interests include digital communications, adaptive digital signal processing and algorithms, and architectures for real-time image formation from synthetic aperture radar. He is currently president of XCAD corporation, where he is directing the development of CAD cools for digital communications, and signal and image processing applications.

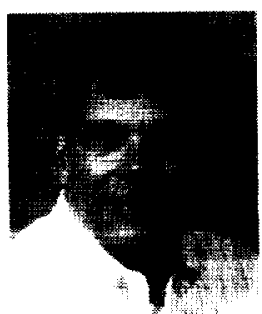

Chase P. Hearn received the B.S.EE degree from North Carolina State University at Raleigh in 1961 and the M.S.EE degree from the University of Virginia in 1968 .

He has been a research engineer with the NASA Langley Research Center since 1961. His work there has involved analysis, design, and development of radio-frequency and microwave circuits and systems. Major projects include a predetection polarization diversity combiner for reception of arbitrarily polarized wideband signals (1969); the Piloted-Aircraft Data System, which provides high-reliability flight and safety data for aircraft flight tests using space and frequency-diversity techniques (1972); a C-band landing guidance system for VTOL aircraft (1978); a Kaband bi-static radar for sea-state measurements (1980); the "Pushbroom" Microwave Radiometer for remote sensing of soil moisture (1982); analysis. design, and fabrication of specialized hybrid microwave integrated circuits (1987); and the Microwave Reflectometer Ionization Sensor for aerobraking research (1992). He is currently working on electrical beam-steering technology and a C-band radiometer for laboratory measurement applications.

$\mathrm{He}$ holds three patents, has two pending, and is a member of Eta Kappa Nu.

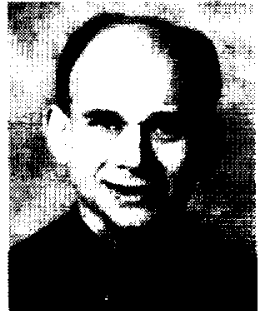

Robert T. Neece received the Ph.D. degrec in electrical engineering from North Carolina State University at Raleigh in 1986.

From 1986 to 1987. he was employed as a project engineer at Microwave Laboratories, Inc., in Raleigh, NC, and from 1987 to 1988, he worked as a microwave systems engineer for PRC at NASA Langley Research Center in Hampton, VA. After becoming a Civil Servant in 1988, he worked at NASA/Langley as the instrument manager for the Microwave Reflectometer Ionization Sensor through 1992. In 1993, he began work developing programs for airborne sensors and enhanced and synthetic vision for commercial aircraft. Dr. Neece is currently the project manager for the development of a sensor for aircraft wake vontices. 